\title{
Complex span tasks, simple span tasks, and cognitive abilities: A reanalysis of key studies
}

\author{
ROBERTO COLOM, IRENE REBOLLO, FRANCISCO J. ABAD, and PEI CHUN SHIH \\ Universidad Autónoma de Madrid, Madrid, Spain
}

\begin{abstract}
There is great interest in the relationships between memory span tasks and cognitive abilities. However, the causes underlying their correlation remain unknown. In the present article, five key data sets were reanalyzed according to two criteria: They must consider complex span tasks (so-called working memory [WM] tasks) and simple span tasks (so-called short-term memory [STM] tasks), and they must comprise cognitive ability measures. The obtained results offer several points of interest. First, memory span tasks should be conceived from a hierarchical perspective: They comprise both general and specific components. Second, the general component explains about four times the variance explained by the specific components. Third, STM and WM measures are closely related. Fourth, STM and WM measures share the same common variance with cognitive abilities. Finally, the strong relationship usually found between memory span tasks and cognitive abilities could be tentatively interpreted by the component shared by STM and WM-namely, the capacity for temporarily preserving a reliable memory representation of any given information.
\end{abstract}

There is increasing interest in the observed relationship between memory span tasks and cognitive abilities (Ackerman, Beier, \& Boyle, 2002, 2005; Bayliss, Jarrold, Gunn, \& Baddeley, 2003; Colom, Abad, Rebollo, \& Shih, 2005; Colom, Flores-Mendoza, \& Rebollo, 2003; Colom, Rebollo, Palacios, Juan-Espinosa, \& Kyllonen, 2004; Colom \& Shih, 2004; Conway, Cowan, Bunting, Therriault, \& Minkoff, 2002; Engle, Tuholski, Laughlin, \& Conway, 1999; Kane et al., 2004; Kyllonen \& Christal, 1990; Lohman, 2000; Miyake, Friedman, Rettinger, Shah, \& Hegarty, 2001; Süß, Oberauer, Wittmann, Wilhelm, \& Schulze, 2002).

Within the investigation of memory span tasks, there are two basic theoretical issues presumably germane to the understanding of individual differences in cognitive abilities (Miyake, 2001). First, to what extent are individual differences in memory span tasks domain specific or domain general? Friedman and Miyake (2000) and Mackintosh and Bennett (2003) highlighted the domain-specific components of complex span tasks, whereas Turner and Engle (1989), Kane et al. (2004), and Colom and Shih (2004) emphasized the domain-general component. Nevertheless, a quantification of the importance of those domain-general and domain-specific components is strongly needed. To our knowledge, this quantification has not yet been attempted.

Engle, Kane, and Tuholski (1999) proposed a hierarchical view based on both domain-general and domain-specific

The research referred to in this article was supported by Grant BSO2002-01455 from the Ministerio Español de Ciencia y Tecnología. We thank Gerald Tehan, Mike Kane, and one anonymous reviewer for their helpful comments. Correspondence concerning this article should be addressed to R. Colom, Facultad de Psicología, Universidad Autónoma de Madrid, 28049 Madrid, Spain (e-mail: roberto.colom@uam.es). components. A general factor can be equated with the domain-general component of span tasks, whereas specific factors can be equated with domain-specific components. This hierarchical view is explicitly tested in the present article. In addition, the relative importance of those components is specifically quantified. This quantification can help to illuminate the causes of the association usually found between memory span tasks and cognitive abilities. Moreover, it could shed light on the factors underlying individual differences in these tasks. Thus, the first goal of the present article is met by this quantification.

The second theoretical issue concerns which factors underlie individual differences in memory span tasks. Surely, there are several relevant factors (Miyake, 2001). However, here we focus on the influential theoretical framework proposed by Engle, Kane, and Tuholski (1999)namely, $\mathrm{WM}=\mathrm{STM}+$ controlled attention. Thus, the second goal of the present article is to test the likelihood of the controlled attention view of the WM system in its relationship to cognitive abilities.

Interestingly, Engle and Kane (2004) distinguished the microanalytic approach from the macroanalytic approach. The former approach is based on the selection of participants according to their scores on a given WM measure. Participants with high and with low scores are grouped and compared in elementary cognitive tasks such as the Stroop, the antisaccade, or the flanker task. The macroanalytic approach, in contrast, tests a large number of participants through several different tasks intended to represent the constructs of interest, such as short-term storage or WM. Structural equation modeling (SEM) analyses are usually performed to test the likelihood of a given theoretical view. Engle and Kane stated that the macroanalytic approach "gives a much cleaner and clearer picture [than 
the microanalytic approach] of WM at the construct level and the degree of relationship of other constructs with WM" (p. 157).

Although the empirical evidence coming from the microanalytic approach is clearly pertinent to the main topic considered here, the present article focuses exclusively on the macroanalytic approach and on exploring several key published data sets addressing the relationships among the constructs of interest - namely, data sets based on complex span tasks (which measure WM), data sets based on simple span tasks (which measure STM), and data sets based on measures of several different cognitive abilities.

\section{SELECTED DATA SETS AND RATIONALE FOR THE REANALYSES}

The data sets of Engle, Tuholski, et al. (1999), Miyake et al. (2001), Conway et al. (2002), Bayliss et al. (2003), and Kane et al. (2004) were selected. The selection was made according to two criteria. First, the studies had to take into account simple and complex span tasks - that is, STM and WM measures, respectively. Some data sets took into account verbal and quantitative measures, others considered spatial measures, and still others used some combination of theses facets of content. Second, the studies had to measure cognitive abilities. This criterion is derived from the necessity to analyze the place of STM, WM, and cognitive abilities within the factor of space (Kline, 1994).

\section{First Goal of the Present Reanalyses}

In order to meet the first goal (i.e., the quantification of the relative contributions of domain-general and domainspecific components of memory span tasks to individual differences in cognitive abilities), we applied a specific exploratory factor analysis (EFA): the Schmid-Leiman (1957) hierarchical factor analysis (Carroll, 1993; Jensen \& Weng, 1994; Loehlin, 2004).

In the Schmid-Leiman hierarchical factor analysis (SLHFA), the higher order factors are allowed to account for as much of the correlation among the observed variables as they can, whereas the lower order factors are reduced to residual factors uncorrelated with each other and with the higher order factors. Therefore, each factor represents the independent contribution of the factor in question (Schmid \& Leiman, 1957). We will discuss one example of how the SLHFA works.

Table 1 shows a correlation matrix submitted to an SLHFA and the resulting hierarchical factor matrix.

The factor matrix presented in Table 1 comprises the loadings of the nine measures both on the higher order factor (general intelligence, or $g$ ) and on the first-order factors $\left(F_{1}, F_{2}\right.$, and $\left.F_{3}\right)$. The commonality $\left(h^{2}\right)$ represents the proportion of the measures' total variance attributable to $g, F_{1}, F_{2}$, and $F_{3}$. It is easy to see that $h^{2}$ results from the sum of the measures' squared factor loadings on $g, F_{1}$, $F_{2}$, and $F_{3}$. The uniqueness $\left(u^{2}\right)$ represents specific variance and error variance. Of course, $h^{2}+u^{2}=1$. Finally, the last row of Table 1 comprises the percentage of the total variance attributable to $g, F_{1}, F_{2}$, and $F_{3}$ (see Jensen, 1998, for further details).

It is usually observed, within the cognitive abilities domain, that the general factor accounts for a larger proportion of the total variance than any other factor and all other factors combined (Carroll, 1993; Jensen, 1998; Lubinski, 2004).

The SLHFA was applied to the selected data sets. First, first-order factors were extracted from the correlation matrix. Factors with eigenvalues greater than 1 are retained for further analyses (Salthouse, Atkinson, \& Berish, 2003). Second, the correlation matrix among first-order factors was subjected to a new factor analysis. Principal axis factoring was employed to extract the factors and was followed by a Promax rotation. Remember that the obtained factors are mutually independent, which is especially important for the pursued quantification.

Each measure has a value in both the higher order and first-order factors. Those values can be used to compute the percentage of variance explained by the second- (general) and first-order (specific) factors. The interpretation is straightforward: The higher the percentage of variance explained, the greater is the relevance of the factor in accounting for the observed individual differences.

It is important to note that the SLHFA is much more appropriate than nonhierarchical factor analyses, such as those computed by Engle, Tuholski, et al. (1999) and Bayliss et al. (2003), because the factors obtained after a nonhierarchical factor analysis confound the shared

Table 1

Correlation Matrix Showing the Result of a Schmid-Leiman (1957) Hierarchical Factor Analysis

\begin{tabular}{|c|c|c|c|c|c|c|c|c|c|c|c|c|c|c|c|}
\hline & V1 & $\mathrm{V} 2$ & V3 & V4 & V5 & V6 & V7 & V8 & V9 & $g$ & $F_{1}$ & $F_{2}$ & $F_{3}$ & $h^{2}$ & $u^{2}$ \\
\hline V1 & & & & & & & & & & .764 & .434 & .008 & .008 & .772 & .228 \\
\hline $\mathrm{V} 2$ & .757 & & & & & & & & & .751 & .378 & .023 & .009 & .707 & .293 \\
\hline V3 & .717 & .680 & & & & & & & & .746 & .356 & .026 & .025 & .685 & .315 \\
\hline V4 & .602 & .608 & .618 & & & & & & & .786 & .035 & .377 & .022 & .762 & .238 \\
\hline V5 & .618 & .627 & .619 & .694 & & & & & & .763 & .100 & .257 & .062 & .662 & .338 \\
\hline V6 & .563 & .558 & .540 & .745 & .678 & & & & & .737 & .020 & .439 & .009 & .736 & .264 \\
\hline V7 & .559 & .568 & .547 & .571 & .573 & .545 & & & & .731 & .005 & .007 & .405 & .698 & .302 \\
\hline V8 & .612 & .599 & .664 & .628 & .589 & .541 & .617 & & & .735 & .169 & .044 & .200 & 611 & .389 \\
\hline V9 & .629 & .612 & .606 & .658 & .668 & .600 & .762 & .673 & & .811 & .003 & .031 & .412 & .828 & .172 \\
\hline Variance & & & & & & & & & & 5.18 & .50 & .41 & .38 & 6.47 & 2.54 \\
\hline$\%$ Variance & & & & & & & & & & 57.5 & 5.5 & 4.5 & 4.2 & 71.7 & 28.3 \\
\hline
\end{tabular}


variance among all measures and the variance specific to groups of measures. The great advantage of the SLHFA is that those distinguishable sources of variance are clearly separated. In order to test the likelihood of the hierarchical view, one statistical hierarchical representation of the corresponding sources of variance is needed, a goal that cannot be achieved through conventional exploratory factor analyses.

The predictions are directly linked to the first theoretical issue already mentioned - namely, the extent to which individual differences are domain specific or domain general. The domain general view predicts the emergence of a very robust higher order factor and negligible first-order factors. The domain specific view predicts a weak higher order factor and strong first-order factors. The hierarchical view predicts a powerful second-order factor (reflecting the domain-general component) and nonnegligible first-order factors (reflecting the domain-specific components).

\section{Second Goal of the Present Reanalyses}

In order to meet the second goal, SEM techniques were applied. The SEM analyses were performed with the AMOS program (Arbuckle, 2003). This program provides several fitting measures, but only some of them are considered here. First, the $\chi^{2} / d f$ index is frequently considered as a rule of thumb because it corrects the high sensitivity of the $\chi^{2}$ statistic for large sample sizes (Jöreskog, 1993). Values showing a good fit must be under 2.0. Second, the root mean square error of approximation (RMSEA) is usually recommended because it is sensitive to misspecification of the model. Values between 0 and .05 indicate a good fit, values between .05 and .08 represent acceptable errors, and values greater than .10 are indicative of a poor fit (Ackerman et al., 2002; Byrne, 1998; Jöreskog, 1993). Finally, the comparative fit index (CFI) is also reported; acceptable values are larger than .90 (Marsh, Balla, \& McDonald, 1988).

The selected data sets allow the testing of the main prediction that can be derived from Engle, Kane, and Tuholski's (1999) model (WM = STM + controlled attention). If this controlled attention model is consistent with the available evidence, then WM must be the primary predictor of the considered cognitive abilities. The structural coefficient of STM over a given cognitive ability is expected to be much smaller (Conway et al., 2002; Engle, Tuholski, et al., 1999; Kane et al., 2004). Furthermore, and especially importantly, the higher predictive power of WM should be detected across data sets.

However, there are both theoretical arguments and empirical evidence against this prediction. First, the theoretical assumption is that memory span tasks predict a given cognitive ability primarily by requiring the subject to temporarily store information. In that vein, Engle, Tuholski, et al. (1999), Conway et al. (2002), and Kane et al. (2004) proposed that the WM system is devoted to keeping memory representations active and accessible. Cowan (2004) defined WM as the system dedicated to the retention of information in a temporarily accessible form.
Second, Embretson (1995) examined the roles of two latent factors that parallel STM and WM (memory load and general control processing, respectively). These factors served as predictors of performance on a test measuring fluid intelligence. The results indicated that both factors predicted individual differences on the fluid test with large values. Third, Colom et al. (2005) analyzed the three-way relationship among general intelligence $(g)$, STM, and $\mathrm{WM}$, finding that STM and WM are equally pertinent to predicting individual differences in $g$.

Importantly, the alternative view does not reject the probable relevance of WM components unrelated to the temporary maintenance of any given information, mainly because they specifically could reflect general control processes (whatever they may be).

Figure 1 shows the basic SEM. This model indicates that a given cognitive ability is predicted by WM and STM defined as latent factors. In order to avoid the recognized multicollinearity problem that characterizes WM and STM measures (Engle, Tuholski, et al., 1999; Kane et al., 2004; Miyake et al., 2001), we took the following approach (see Conway et al., 2002, p. 176): WM tasks place great demands on STM, but (theoretically) WM tasks require the simultaneous processing and storage of information. Therefore, in order to compare the relative contributions of STM and WM to cognitive abilities, we specified all the memory span tasks as indicators of STM, but only WM measures as indicators of WM.

Researchers have used different approaches to deal with this multicollinearity problem, and their views vary across studies. We have some reservations about this practice and find it much more useful to analyze different data sets against the same basic model.

In their very impressive study, Kane et al. (2004) addressed the relative contributions of WM, verbal STM, and visuospatial STM to the relation between memory span and cognitive ability. Their general model consisted of an executive attention factor, with loadings from all their memory variables, presumably reflecting the domain-general executive variance shared by all the memory span tasks. The model also included domain-specific factors, with loadings from the verbal and spatial tasks on the storageverbal and storage-spatial factors, respectively. Kane et al. proposed that the common variance among memory span tasks reflects executive rather than storage processes.

However, in two key previous studies, Engle, Tuholski, et al. (1999) and Conway et al. (2002) proposed that the common variance between WM and STM reflects primarily storage, whereas the residual WM variance reflects primarily executive control processes. Engle, Tuholski, et al. allowed a free correlation between their STM and WM latent factors, whereas Conway et al. treated the multicollinearity problem using the basic model depicted in Figure 1. Therefore, no fewer than three different operational approaches were employed in those studies.

It is reasonable to state that Kane et al.'s (2004) general executive factor is a complex mixture of short-term storage and general control processes (whatever they may 


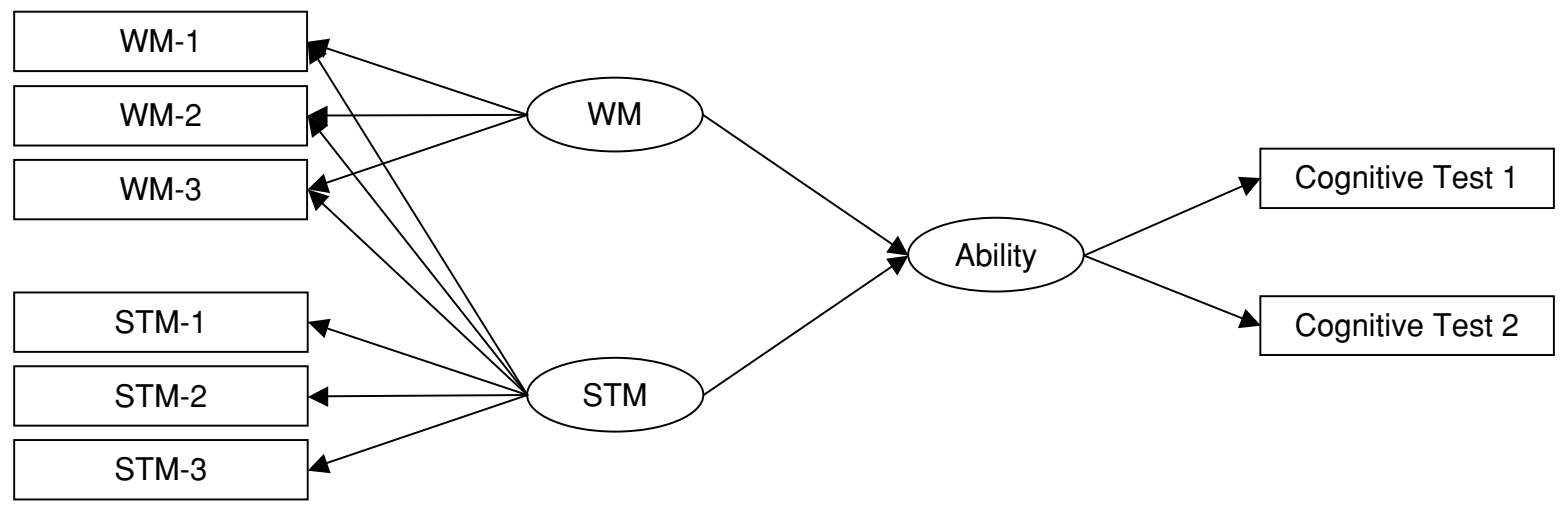

Figure 1. Basic structural equation modeling. A given cognitive ability is predicted by working memory (WM) and short-term memory (STM), defined as latent factors.

be). Theoretically, it is hard to admit that their general factor appropriately represents mainly the presumed general control processes underlying all their memory span tasks. In order to favor our general approach, it is imperative to note that their STM tasks were carefully designed to measure mainly short-term storage:

... we choose clarity of interpretation over breadth of measurement, and modelled all the verbal and spatial WM tasks after reading span ... [P] erformance in these tasks, with either verbal or visuo-spatial materials, reflects one's ability to encode, maintain, and retrieve lists of isolated stimuli in the face of a regularly occurring, highly interfering distractor task. . . Y Yet an additional benefit of the span procedure is that we could create STM versions of each of our WM tasks that presented the same to-beremembered stimuli, but without the additional processing demand of the secondary task. (p. 193, emphasis added)

We think these are strong reasons to state that it is more theoretically driven and conservative to assume that Kane et al.'s (2004) general memory span factor more appropriately represents mainly the short-term storage component undoubtedly shared by all their memory span tasks. Their STM tasks presented the same to-be-remembered stimuli as their WM tasks, but only the latter comprised additional processing demands from a secondary task. Thus, a latent factor defined only by their WM tasks should represent mainly the additional processing requirements (with the storage component partialed out).

In summary, it is risky to assume that a latent factor clearly mixing storage plus processing can be seen primarily as a clear-cut representation of the central executive. What we find much more compelling is the view that all memory span tasks share a storage component, whereas WM tasks entail additional processing requirements (whatever they may be). Furthermore, it is strange to state that sometimes all memory span tasks share their storage component and sometimes they are all presumed to share their executive component (especially when the STM tasks were explicitly designed to measure mainly storage; see Kane et al., 2004, p. 193).
Given this state of affairs, we propose that the general SEM depicted in Figure 1 could be an appropriate way to test the relative contributions of STM and WM to the prediction of a given cognitive ability. If the controlled attention model fits the cumulative data, then the predictive power of WM is expected to be much greater than that of STM across data sets. If this proves to be the case, then the controlled attention view endorsed by Engle, Kane, and Tuholski (1999) must be seen as consistent with the available empirical evidence. However, if this is not the case, then one alternative (and more parsimonious) view should be considered seriously: The predictive power of memory span measures could come primarily (although not exclusively) from the component shared by STM and WM measures - namely, the capacity to temporarily preserve a reliable memory representation of any given information.

\section{RESULTS}

\section{EFA Results}

EFA findings are presented separately for each data set. The broad theoretical derivations that can be extracted from these results are discussed in the General Discussion section.

Engle, Tuholski, et al.'s (1999) data set. Sixteen measures were analyzed (see Table 2). The correlation matrix can be found in Engle, Tuholski, et al. (1999, p. 319). Table 2 displays the hierarchical factor matrix obtained from the reanalysis of the present data set.

The first-order factor analysis produced four factors. The first factor was loaded by WM and STM measures, the second factor by cognitive ability measures, and the third and fourth factors by the remaining span measures. Note that WM and STM measures are not separated into distinguishable first-order factors.

The results include several points of interest. (1) The second-order factor accounts for $26.81 \%$ of the variance. (2) The general factor explains more variance than do any of the first-order factors. (3) The average loading in the second-order factor is .532 for complex span or WM 
Table 2

Schmid-Leiman (1957) Hierarchical Factor Matrix Obtained From Engle, Tuholski, et al.'s (1999) Data Set

\begin{tabular}{lccccc}
\multicolumn{1}{c}{ Task } & $g$ & $F_{1}$ & $F_{2}$ & $F_{3}$ & $F_{4}$ \\
\hline \multicolumn{5}{c}{ Working Memory (WM) Measures } \\
OSpan & .602 & .302 & .114 & .035 & .123 \\
RSpan & .470 & .257 & .034 & .039 & .041 \\
CSpan & .526 & .191 & .126 & .136 & .041 \\
\multicolumn{5}{c}{ Short-Term Memory Measures } \\
BSpan & .531 & .258 & .065 & .267 & .028 \\
FSpanD & .613 & .392 & .227 & .206 & .039 \\
FSpanS & .575 & .319 & .065 & .205 & .019 \\
& \multicolumn{5}{c}{ Other WM Measures } \\
KTrack & .459 & .168 & .188 & .003 & .089 \\
IFRSM & .479 & .486 & .026 & .086 & .203 \\
IFRPM & .414 & .126 & .035 & .228 & .497 \\
ABCD & .522 & .042 & .232 & .468 & .129 \\
ContOp & .552 & .072 & .126 & .376 & .036 \\
Rand & .230 & .019 & .052 & .130 & .407 \\
& \multicolumn{5}{c}{ Cognitive Measures } \\
Cattell & .386 & .075 & .760 & .094 & .172 \\
Raven & .478 & .038 & .615 & .099 & .009 \\
VSAT & .626 & .229 & .328 & .041 & .096 \\
QSAT & .659 & .104 & .507 & .042 & .193 \\
\% Variance & 26.81 & 5.43 & 9.5 & 4 & 3.62 \\
\hline
\end{tabular}

Note-OSpan, operation span; RSpan, reading span; CSpan, counting span; BSpan, backward span; FSpanD, forward span dissimilar; FSpanS, forward span similar; KTrack, keeping track; IFRSM, immediate freerecall secondary memory; IFRPM, immediate free-recall primary memory; ContOp, continuous opposites; Rand, random number generation; Cattell, Culture Fair Intelligence Test; Raven, Standard Progressive Matrices Test; VSAT, Verbal Scholastic Aptitude Test; QSAT, Quantitative Scholastic Aptitude Test.

measures, .573 for simple span or STM measures, and .537 for the measures of cognitive ability. This suggests that complex and simple span tasks share the same common variance with cognitive abilities. (4) There is not one first-order factor for WM measures and another for STM measures-both measures load in the same first-order factor $\left(F_{1}\right)$. Taken together, these results strongly fit the prediction of the hierarchical view.

Miyake et al.'s (2001) data set. Twelve measures were considered (see Table 3 ). The correlation matrix can be found in Miyake et al. (2001, p. 163). Table 3 displays the hierarchical factor matrix obtained from the reanalysis of the present data set.

The first-order factor analysis produced three factors. The first factor was loaded by complex (WM) and simple (STM) span measures, and the second and third factors were loaded by cognitive ability measures. Note that WM and STM measures are not separated into distinguishable first-order factors.

The results include several points of interest. (1) The second-order factor accounts for $27.83 \%$ of the variance. (2) The general factor explains more variance than do any of the first-order factors. (3) The average loading in the second-order factor is .507 for complex span or WM measures, .481 for simple span or STM measures, and .575 for the measures of cognitive ability. This suggests that WM and STM share the same common variance with cognitive abilities. (4) There is not one first-order factor for WM measures and another for STM measures - both measures load in the same first-order factor $\left(F_{1}\right)$. Taken together, these results strongly fit the prediction of the hierarchical view.

Conway et al.'s (2002) data set. Thirteen measures were analyzed (see Table 4). The correlation matrix can be found in Conway et al. (2002, p. 173). Table 4 displays the hierarchical factor matrix obtained from the reanalysis of the present data set.

The first-order factor analysis produced three factors. The first factor was loaded by complex (WM) and simple (STM) span measures, the second factor by processing speed measures, and the third factor by cognitive ability measures. Note that WM and STM measures are not separated into distinguishable first-order factors.

The results include several points of interest. (1) The second-order factor accounted for $19.15 \%$ of the variance. (2) The general factor explains more variance than do any of the first-order factors. (3) The average loading in the second-order factor is .560 for complex span or WM measures, .528 for simple span or STM measures, and .302 for the measures of cognitive ability. This suggests that WM and STM share the same common variance with cognitive abilities. (4) There is not one first-order factor for WM measures and another for STM measures - both measures load in the same first-order factor $\left(F_{1}\right)$. Taken together, these results fit the prediction of the hierarchical view.

Bayliss et al.'s (2003) data set. Although Bayliss et al. reported two experiments, only the first is considered in

Table 3

Schmid-Leiman (1957) Hierarchical Factor Matrix Obtained From Miyake et al.'s (2001) Data Set

\begin{tabular}{|c|c|c|c|c|}
\hline Task & $g$ & $F_{1}$ & $F_{2}$ & $F_{3}$ \\
\hline \multicolumn{5}{|c|}{ Executive Measures } \\
\hline Hanoi & .452 & .085 & .171 & .141 \\
\hline Rand & .273 & .086 & .083 & .079 \\
\hline \multicolumn{5}{|c|}{ Working Memory Measures } \\
\hline Let rotation & .502 & .353 & .226 & .152 \\
\hline Dot matrix & .512 & .541 & .036 & .054 \\
\hline \multicolumn{5}{|c|}{ Short-Term Memory Measures } \\
\hline Corsi & .439 & .483 & .127 & .164 \\
\hline Dot memory & .524 & .434 & .065 & .023 \\
\hline \multicolumn{5}{|c|}{ Cognitive Measures } \\
\hline Folding & .701 & .046 & .484 & .012 \\
\hline Relat & .726 & .100 & .463 & .187 \\
\hline Card & .560 & .036 & .028 & .507 \\
\hline Flags & .613 & .007 & .042 & .586 \\
\hline Id Pict & .370 & .128 & .083 & .138 \\
\hline Hidden & .480 & .303 & .014 & .180 \\
\hline$\%$ Variance & 27.83 & 8.16 & 4.75 & 6.41 \\
\hline
\end{tabular}

Note-Hanoi, Tower of Hanoi; Rand, random number generation; Let rotation, letter rotation; Corsi, Corsi block; Folding, paper folding; Relat, space relations; Card, card rotation; Id Pict, identical pictures; Hidden, hidden patterns. 
Table 4 Schmid-Leiman (1957) Hierarchical Factor Matrix Obtained From Conway et al.'s (2002) Data Set

\begin{tabular}{|c|c|c|c|c|}
\hline Task & $g$ & $F_{1}$ & $F_{2}$ & $F_{3}$ \\
\hline \multicolumn{5}{|c|}{ Working Memory Measures } \\
\hline OSpan & .577 & .384 & .011 & .126 \\
\hline RSpan & .556 & .370 & .006 & .125 \\
\hline CSpan & .547 & 286 & .046 & .358 \\
\hline \multicolumn{5}{|c|}{ Short-Term Memory Measures } \\
\hline STMu & .570 & .498 & .041 & .129 \\
\hline STMf & .491 & .460 & .148 & .103 \\
\hline STMus & .549 & 399 & .179 & .091 \\
\hline STMfs & .502 & .335 & .107 & .031 \\
\hline \multicolumn{5}{|c|}{ Processing Speed (PS) Measures } \\
\hline PS letter & .290 & .014 & .771 & .004 \\
\hline S pattern & .321 & .009 & .768 & .042 \\
\hline S digit & .236 & .019 & .575 & .041 \\
\hline S Dig Sym & .062 & .013 & .018 & .071 \\
\hline \multicolumn{5}{|c|}{ Cognitive Measures } \\
\hline Cattell & .346 & .017 & .038 & .667 \\
\hline Raven & .259 & .065 & .043 & .685 \\
\hline$\%$ Variance & 19.15 & 8.46 & 12.23 & 8.61 \\
\hline \multicolumn{5}{|c|}{$\begin{array}{l}\text { Note-OSpan, operation span; RSpan, reading span; CSpan, counting } \\
\text { pan; STMu, short-term memory (STM) with unlimited pool of words; } \\
\text { STMf, STM with limited pool of words; STMus, STM with unlimited } \\
\text { oool of words and with articulatory suppression; STMfs, STM with lim- } \\
\text { ted pool of words and with articulatory suppression; PS letter, letter } \\
\text { comparison; PS pattern, pattern comparison; PS digit, digit and letter } \\
\text { copying; PS Dig Sym, digit symbol substitution; Cattell, Culture Fair } \\
\text { Intelligence Test; Raven, Standard Progressive Matrices Test. }\end{array}$} \\
\hline
\end{tabular}

the present reanalysis. In that experiment, a sample of 75 children was considered; such a sample can be seen as representative of the population, which is highly desirable. Furthermore, the sample of their second experiment comprised only 48 participants, who were undergraduate university students. It is well-known that with small samples the correlation coefficient is highly unreliable (Detterman, 1989). Therefore, only their first sample was considered here.

Eleven measures were considered (see Table 5). The correlation matrix can be found in Bayliss et al. (2003, p. 78). Table 5 displays the hierarchical factor matrix obtained from the reanalysis of the present data set.

The first-order factor analysis produced four factors. The first factor was loaded by cognitive ability measures, the second factor by processing measures, and the third and fourth factors by complex span (WM) and simple span (STM) measures. Note that WM and STM measures are not separated into distinguishable first-order factors.

The results include several points of interest. (1) The second-order factor accounts for $31.9 \%$ of the variance. (2) The general factor explains more variance than do any of the first-order factors. (3) The average loading in the second-order factor is .523 for complex span or WM measures, .510 for simple span or STM measures, and .644 for the measures of cognitive ability. This suggests that WM and STM share the same common variance with cognitive abilities. (4) There is not one first-order factor for WM measures and another for STM measures $-F_{3}$ and $F_{4}$ are loaded by both measures. Taken together, these results strongly fit the prediction of the hierarchical view.

Kane et al.'s (2004) data set. Twenty-five measures were considered (see Table 6). The correlation matrix can be found in Kane et al. (2004, p. 201). Table 6 displays the hierarchical factor matrix obtained from the reanalysis of the present data set.

The first-order factor analysis produced three factors. The first factor was loaded by all the cognitive ability measures and the spatial STM tasks (no WM task here), the second factor was loaded by all the WM measures plus the spatial STM measures, and the third factor was loaded by five of the six verbal memory span measures plus verbal reasoning tests. Note that WM and STM measures are not separated into distinguishable first-order factors.

The results include several points of interest. (1) The second-order factor accounts for $33.6 \%$ of the variance. (2) The general factor explains more variance than do any of the first-order factors. (3) The average loading in the second-order factor is .634 for complex span or WM measures, .612 for simple span or STM measures, and .533 for the measures of cognitive ability. This suggests that WM and STM share the same common variance with cognitive abilities. (4) There is not one first-order factor for WM measures and another for STM measures. Taken together, these results strongly fit the prediction of the hierarchical view.

Table 5

Schmid-Leiman (1957) Hierarchical Factor Matrix Obtained From Bayliss et al.'s (2003) Data Set

\begin{tabular}{|c|c|c|c|c|c|}
\hline Task & $g$ & $F_{1}$ & $F_{2}$ & $F_{3}$ & $F_{4}$ \\
\hline \multicolumn{6}{|c|}{ Working Memory Measures } \\
\hline CSpan Ver Ver & .635 & .066 & .305 & .277 & .056 \\
\hline CSpan Ver Vis & .565 & .120 & .171 & .153 & .352 \\
\hline CSpan Vis Ver & .476 & .161 & .143 & .482 & .003 \\
\hline CSpan Vis Vis & .418 & .001 & .032 & .076 & .405 \\
\hline \multicolumn{6}{|c|}{ Short-Term Memory (STM) Measures } \\
\hline STM digit span & .467 & .103 & .044 & .591 & .007 \\
\hline STM Corsi & .554 & .175 & .154 & .093 & .375 \\
\hline \multicolumn{6}{|c|}{ Processing Speed Measures } \\
\hline PS Ver & .559 & .039 & .762 & .079 & .033 \\
\hline PS Vis & .551 & .037 & .580 & .004 & .026 \\
\hline \multicolumn{6}{|c|}{ Cognitive Measures } \\
\hline Raven & .618 & .527 & .001 & .144 & .210 \\
\hline Reading & .636 & .695 & .049 & .119 & .169 \\
\hline Math & .680 & .562 & .072 & .023 & .083 \\
\hline$\%$ Variance & 31.9 & 10.63 & 9.9 & 6.72 & 4.63 \\
\hline
\end{tabular}

Note - CSpan Ver Ver, verbal processing and verbal storage; CSpan Ver Vis, verbal processing and visuospatial storage; CSpan Vis Ver, visuospatial processing and verbal storage; CSpan Vis Vis, visuospatial processing and visuospatial storage; STM digit span, short-term memory (STM) digit span; STM Corsi, STM Corsi block; PS Ver, verbal processing speed; PS Vis, visual processing speed; Raven, Colored Progressive Matrices Test; Reading, reading achievement test; Math, math achievement test. 
Table 6

Schmid-Leiman (1957) Hierarchical Factor Matrix Obtained From Kane et al.'s (2004) Data Set

\begin{tabular}{|c|c|c|c|c|}
\hline Measure & $g$ & $F_{1}$ & $F_{2}$ & $F_{3}$ \\
\hline \multicolumn{5}{|c|}{ Verbal Working Memory (WM) } \\
\hline OpeSpan & .646 & .098 & .307 & .385 \\
\hline ReadSpan & .647 & .046 & .310 & .328 \\
\hline CouSpan & .519 & .141 & .441 & .124 \\
\hline \multicolumn{5}{|c|}{ Spatial WM } \\
\hline NavSpan & .611 & .053 & .508 & .072 \\
\hline SymmSpan & .675 & .090 & .433 & .052 \\
\hline RotaSpan & .704 & .118 & .459 & .020 \\
\hline \multicolumn{5}{|c|}{ Verbal Short-Term Memory (STM) } \\
\hline WordSpan & .567 & .088 & .098 & .562 \\
\hline LettSpan & .609 & .015 & .120 & .500 \\
\hline DigSpan & .555 & .113 & .130 & .534 \\
\hline \multicolumn{5}{|c|}{ Spatial STM } \\
\hline BallSpan & .643 & .239 & .313 & .016 \\
\hline ArrwSpan & .684 & .242 & .320 & .047 \\
\hline MatxSpan & .615 & .227 & .318 & .006 \\
\hline \multicolumn{5}{|c|}{ Verbal Reasoning } \\
\hline Inference & .535 & .281 & .056 & .335 \\
\hline Analogy & .571 & .415 & .139 & .336 \\
\hline ReadComp & .562 & .318 & .095 & .374 \\
\hline RemoAsso & .324 & .275 & .158 & .250 \\
\hline Syllogisms & .409 & .355 & .112 & .196 \\
\hline \multicolumn{5}{|c|}{ Visualization } \\
\hline SpaceRel & .587 & .618 & .045 & .100 \\
\hline RotaBlock & .508 & .514 & .074 & .110 \\
\hline SurfDev & .594 & .531 & .046 & .001 \\
\hline FormBrd & .547 & .505 & .106 & .100 \\
\hline PapFold & .534 & .529 & .055 & .074 \\
\hline \multicolumn{5}{|c|}{ Gf } \\
\hline Raven & .541 & .474 & .044 & .006 \\
\hline WASI & .553 & .392 & .042 & .109 \\
\hline Beta & .603 & .373 & .058 & .160 \\
\hline$\%$ Variance & 33.6 & 11.1 & 5.9 & 6.7 \\
\hline
\end{tabular}

Note-OpeSpan, operation span; ReadSpan, reading span; CouSpan, counting span; NavSpan, navigation span; SymmSpan, symmetry span; RotaSpan, rotation span; WordSpan, word span; LettSpan, letter span; DigSpan, digit span; BallSpan, ball span; ArrwSpan, arrow span; MatxSpan, matrix span; Inference, Educational Testing Service (ETS) inference test; Analogy, Air Force Officer Qualifying (AFOQT) analogies test; ReadComp, AFOQT reading comprehension test; RemoAsso, remote associates test; Syllogisms, ETS nonsense syllogisms test; SpaceRel, Differential Aptitude Test (DAT) space relations test; RotaBlock, AFOQT rotated blocks test; SurfDev, ETS surface development test; FormBrd, ETS form board test; PapFold, ETS paper folding test; Gf, fluid intelligence; Raven, Advanced Progressive Matrices Test; WASI, Wechsler Abbreviated Scale of Intelligence; Beta, Revised Beta Examination.

\section{SEM Results}

In order to proceed with the SEM analyses, it is important to keep in mind that, although the already reported EFA analyses suggest that STM and WM are not clearly distinguishable when those constructs are studied along with measures of cognitive ability, they are not identical. In fact, we stated above that reported SEMs used to study the three-way relationship among STM, WM, and cognitive ability usually suffer from the multicollinearity problem, because STM and WM are very closely re- lated. Nevertheless, "closely related" does not imply a perfect correlation. Given that it is widely assumed that STM tasks comprise mainly storage whereas WM tasks comprise storage plus additional processing, it would be interesting to know if that difference makes a significant contribution when both constructs are allowed to predict a given cognitive ability (see Figure 1).

Several SEMs were tested in the present reanalyses. The first 2 were derived from Engle, Tuholski, et al.'s (1999) study; the 3rd, 4th, and 5th were derived from Miyake et al.'s (2001) study; the 6th, from Conway et al.'s (2002) study; the 7th, from Bayliss et al.'s (2003) data set. Finally, the 8th, 9th, and 10th models were derived from Kane et al.'s (2004) data set.

It is important to note at this point that all these data sets are analyzed against the same basic model. This is highly desirable given that different operational approaches (basic models) were used in the cited studies, so the consistency of their results cannot be assessed clearly. For instance, it is difficult to know if the controlled attention view endorsed by Engle, Tuholski, et al. (1999), Conway et al. (2002), and Kane et al. (2004) is supported by the researchers' own empirical data or by their particular analytic approach.

Engle, Tuholski, et al.'s (1999) data set: Fluid intelligence (Gf) and crystallized intelligence (Gc). Engle, Tuholski, et al. measured Gf and Gc as well as WM and STM (see the EFA Results section, above). Therefore, it is possible to test the predictive power of WM and STM over these cognitive abilities.

Figure 2 displays the SEM for Gf. The model fit was $\chi^{2}(15)=18.96, \chi^{2} / d f=1.26, \mathrm{CFI}=.99, \mathrm{RMSEA}=$ .045 (range, .00-.09).

The results show that the structural coefficients of STM and WM over Gf are very similar (.31 and .38, respectively). When both coefficients were constrained to be equal, the result produced a nonsignificant change of fit $\left[\chi^{2}(1)=0.138, p=.710\right]$. Therefore, individual differences in fluid intelligence were predicted by both STM and WM.

Figure 3 displays the SEM for Gc. The model fit was $\chi^{2}(15)=23.27, \chi^{2} / d f=1.55, \mathrm{CFI}=.98, \mathrm{RMSEA}=$ .065 (range, .00-.11).

The results show that the structural coefficient of STM over Gc is greater than that of WM (.65 and .29, respectively). When both coefficients were constrained to be equal, the result produced a significant change of fit $\left[\chi^{2}(1)=5.132, p=.023\right]$. Therefore, individual differences in crystallized intelligence are better predicted by STM than by WM.

Miyake et al.'s (2001) data set: Spatial visualization (SV), spatial relations (SR), and perceptual speed (PS). Miyake et al. measured SV, SR, and PS as well as WM and STM (see the EFA Results section). Therefore, it is possible to test the predictive power of WM and STM over these cognitive abilities.

The SEM for SV did not show an acceptable solution, and therefore it is not discussed here. 


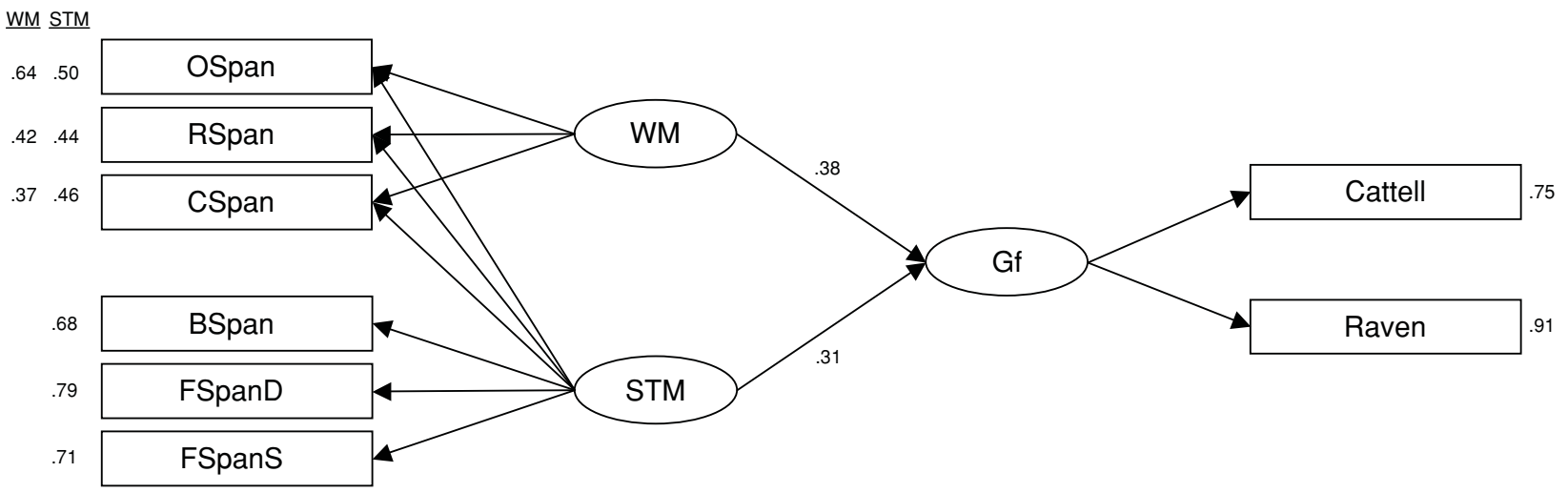

Figure 2. Structural equation modeling for fluid intelligence (Gf) from Engle, Tuholski, et al.'s (1999) data set, with structural coefficients. For meanings of the abbreviations, see the note to Table 2.

Figure 4 displays the SEM for SR. The model fit was $\chi^{2}(5)=3.49, \chi^{2} / d f=0.70$, CFI $=1$, RMSEA $=.00$ (range, .00-.09).

The results show that the structural coefficient of STM over SR is much greater than that of WM (.58 and - 09 [n.s.], respectively). When both coefficients were constrained to be equal, the result produced a significant change of fit $\left[\chi^{2}(1)=9.483, p=.002\right]$. Therefore, individual differences in spatial relations are much better predicted by STM than by WM.

Figure 5 displays the SEM for PS. The model fit was $\chi^{2}(5)=2.97, \chi^{2} / d f=0.59$, CFI $=1$, RMSEA $=.00$ (range, .00-.08).

The results show that the structural coefficient of STM over PS is much greater than that of WM (.67 and .04 [n.s.], respectively). When both coefficients were constrained to be equal, the result produced a significant change of fit $\left[\chi^{2}(1)=10.684, p=.001\right]$. Therefore, individual differences in perceptual speed are much better predicted by STM than by WM.

Conway et al.'s (2002) data set: Gf. Conway et al. measured Gf in addition to WM and STM (see the EFA
Results section). Therefore, it was possible to test the predictive power of WM and STM over this cognitive ability.

Figure 6 displays the SEM for Gf. The model fit was $\chi^{2}(21)=36.64, \chi^{2} / d f=1.74, \mathrm{CFI}=.96, \mathrm{RMSEA}=.08$ (range, .032-.12).

The results show that the structural coefficient of WM over Gf is greater than that of STM (.62 and .21 [n.s.], respectively]. However, when both coefficients were constrained to be equal, the result produced a nonsignificant change of fit $\left[\chi^{2}(1)=3.027, p=.08\right]$. Therefore, individual differences in fluid intelligence can be predicted by both WM and STM.

Bayliss et al's (2003) data set: Reading and math. Two measures of Gc were considered in the present data set. Bayliss et al. also measured STM and WM. We tested the predictive power of those constructs over Gc, but the corresponding SEM did not show an acceptable solution and, therefore, it is not discussed here.

Kane et al.'s (2004) data set: Gf, Gc, and spatial ability (Gv). Three measures of Gf, five measures of Gc, and five measures of Gv were considered in the present data set. Kane et al. also measured STM and WM. There-

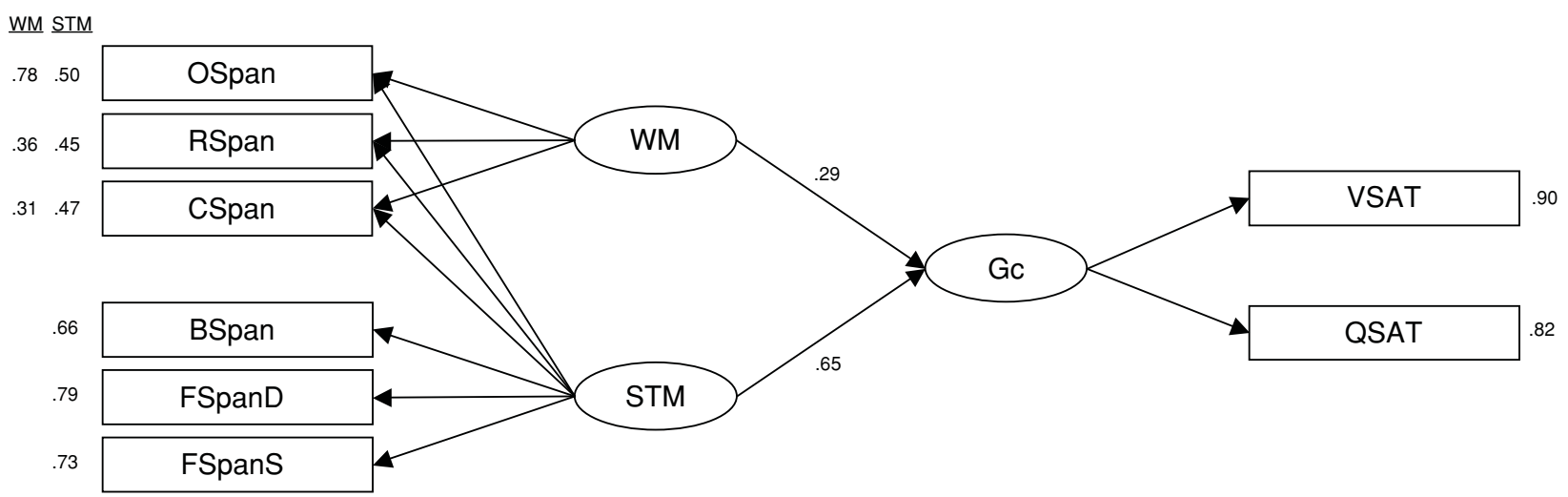

Figure 3. Structural equation modeling for crystallized intelligence (Gc) from Engle, Tuholski, et al.'s (1999) data set, with structural coefficients. For meanings of the abbreviations, see the note to Table 2. 


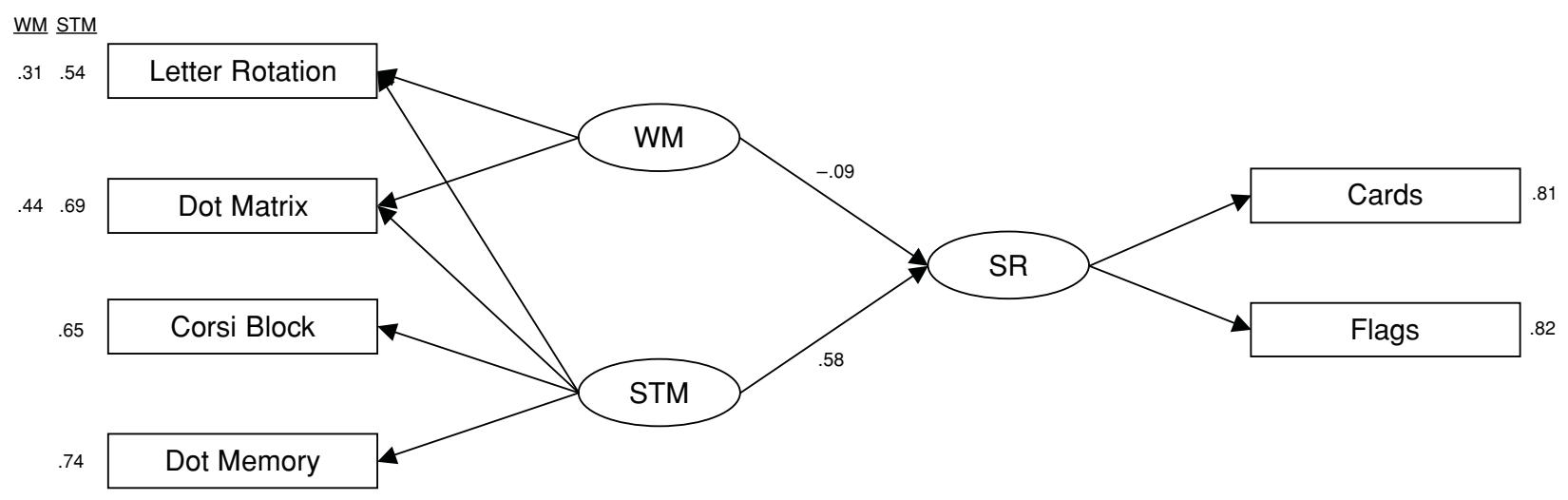

Figure 4. Structural equation modeling for spatial relations (SR) from Miyake et al.'s (2001) data set, with structural coefficients.

fore, it was possible to test the predictive power of WM and STM over Gf, Gc, and Gv.

Figure 7 displays the SEM for Gf. The model fit was $\chi^{2}(78)=176.49, \chi^{2} / d f=2.26, \mathrm{CFI}=.96, \mathrm{RMSEA}=$ .073 (range, .059-.088).

The results depicted in Figure 7 show that the structural coefficient of STM is large (.81), whereas that of WM over Gf is -.09 (n.s.). When both coefficients were constrained to be equal, the result produced a significant change of fit $\left[\chi^{2}(1)=66.520, p=.000\right]$. Thus, STM behaves as a better predictor of Gf than WM.

Figure 8 displays the SEM for Gc. The model fit was $\chi^{2}(107)=209.15, \chi^{2} / d f=1.95, \mathrm{CFI}=.96, \mathrm{RMSEA}=$ .064 (range, .051-.076).

The results depicted in Figure 8 show that the structural coefficient of STM is large (.69), whereas the structural coefficient of WM over Gc is - 07 (n.s.). When both coefficients were constrained to be equal, the result produced a significant change of fit $\left[\chi^{2}(1)=43.393, p=.000\right]$. Thus, STM is a better predictor of Gc than WM.

Finally, Figure 9 displays the SEM for Gv. The model fit was $\chi^{2}(107)=205.07, \chi^{2} / d f=1.92$, RMSEA $=.062$ (range, .049-.075).
The results depicted in Figure 9 show that the structural coefficient of STM is large (.77), whereas the structural coefficient of WM over Gc is -.13 (n.s.). When both coefficients were constrained to be equal, the result produced a significant change of fit $\left[\chi^{2}(1)=73.820\right.$, $p=.000]$. Thus, STM is a better predictor of $\mathrm{Gv}$ than is WM.

\section{GENERAL DISCUSSION}

The main finding derived from the EFA analyses suggests that memory span measures must be considered from a hierarchical point of view. The obtained values for the general and first-order factors can be used to deliver a tentative quantification of the importance of general and specific components: The general component accounts for $20 \%-30 \%$ of the variance, whereas $7 \%$ of the variance is accounted for by specific components linked to both complex and simple memory span measures. This means that the general component is about four times more important than the specific components. The theoretical implication is that individual differences in these tasks are strongly explained by some general component, whereas

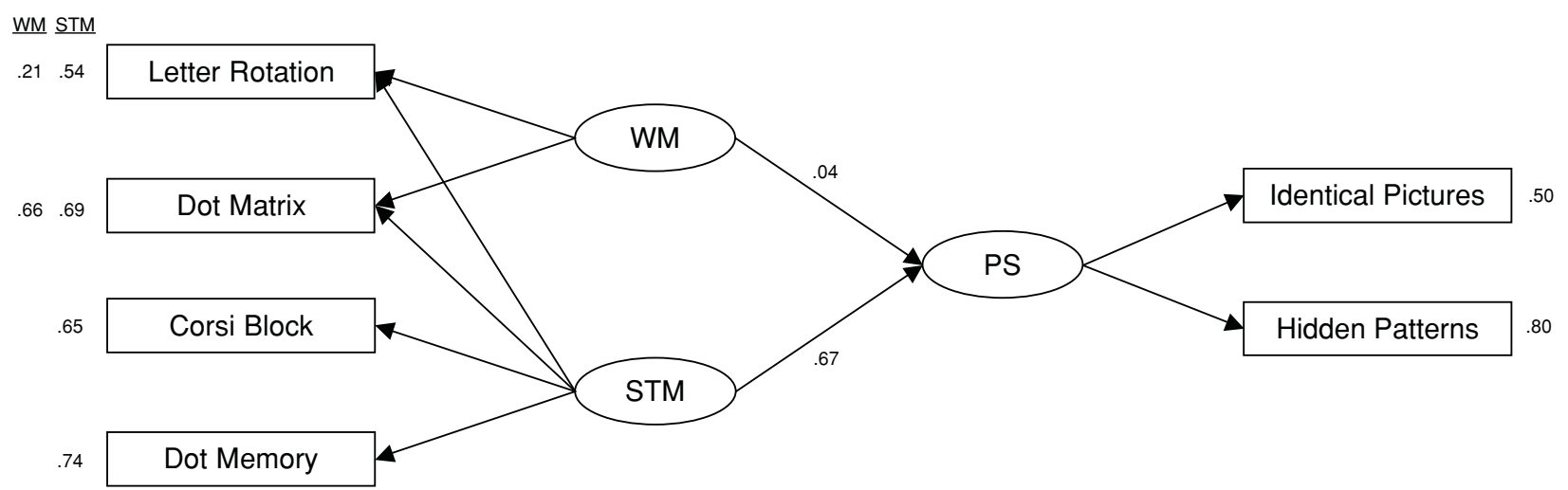

Figure 5. Structural equation modeling for perceptual speed (PS) from Miyake et al.'s (2001) data set, with structural coefficients. 


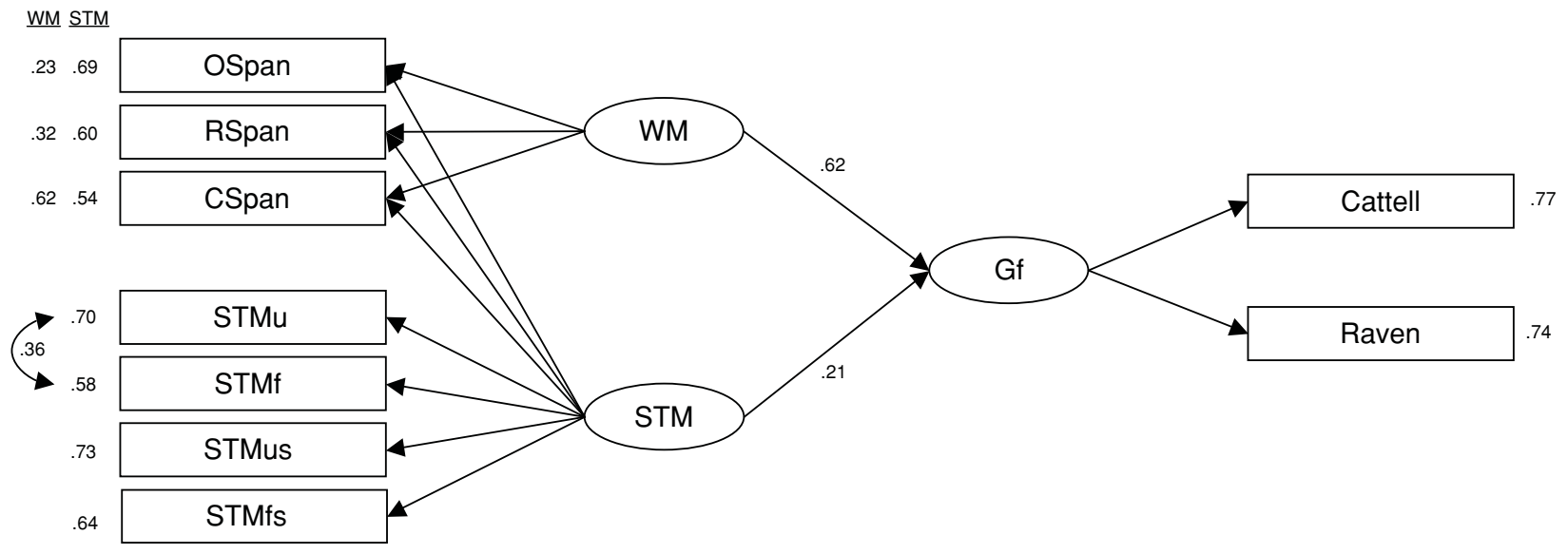

Figure 6. Structural equation modeling for fluid intelligence (Gf) from Conway et al.s (2002) data set, with structural coefficients. For meanings of the abbreviations, see the note to Table 4.

the contribution of specific components is much less pertinent, although not negligible.

Still another important theoretical implication is that complex span measures (of WM) must not be clearly distinguished from simple span measures (of STM). Both measures share something in common that could produce their association with cognitive ability measures. First, the five data sets converge in that WM and STM meas- ures load with one equivalent magnitude in the general (higher order) factor. Second, they load in the same firstorder factor, not in separate first-order factors. If WM and STM measures are not distinguishable by their loadings in the higher order factor, then it seems parsimonious to endorse the view that their shared component (namely, the requirement of temporarily preserving a reliable memory representation of any given information) could be con-

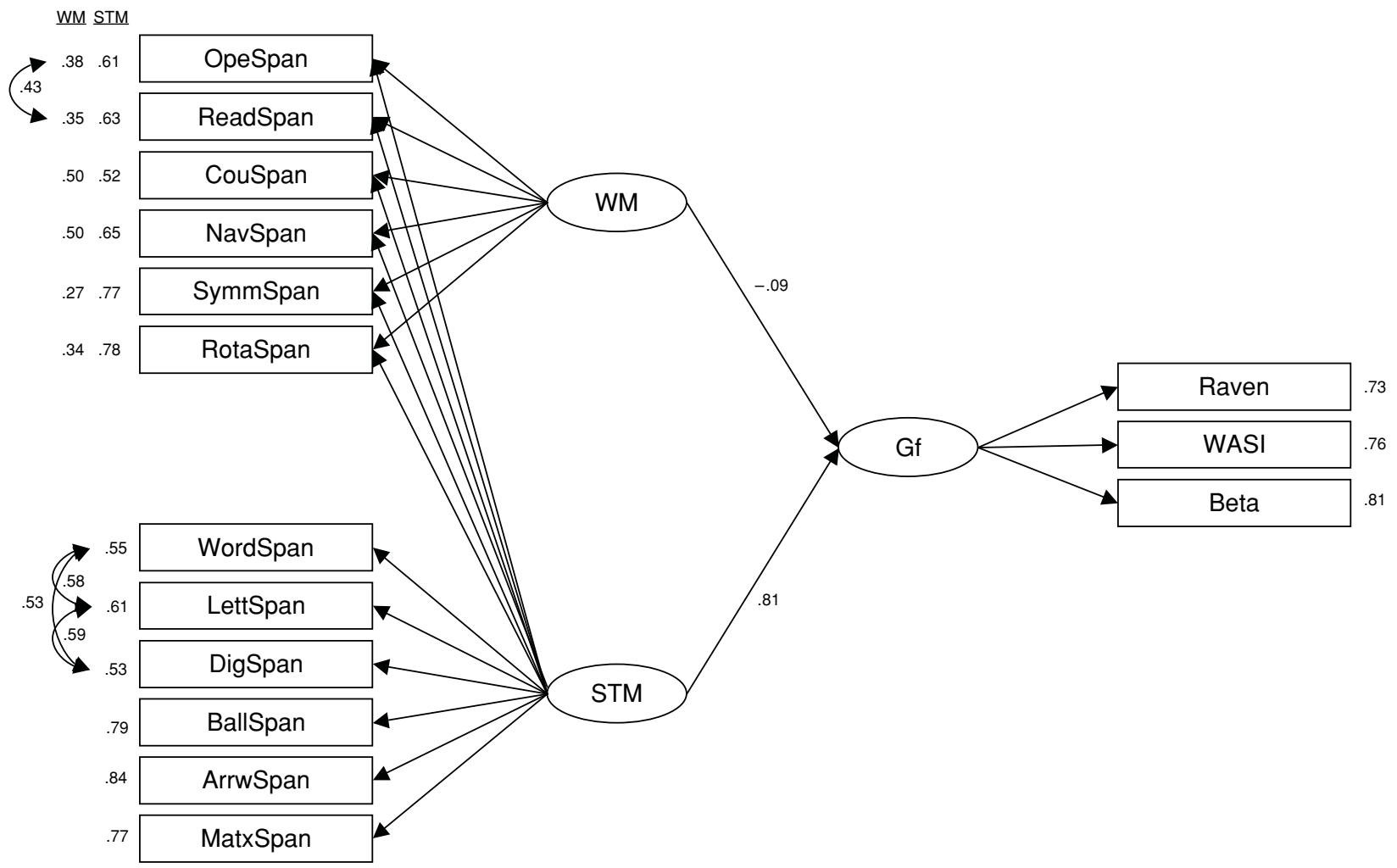

Figure 7. Structural equation modeling for fluid intelligence (Gf) from Kane et al.'s (2004) data set, with structural coefficients. For meanings of the abbreviations, see the note to Table 6. 


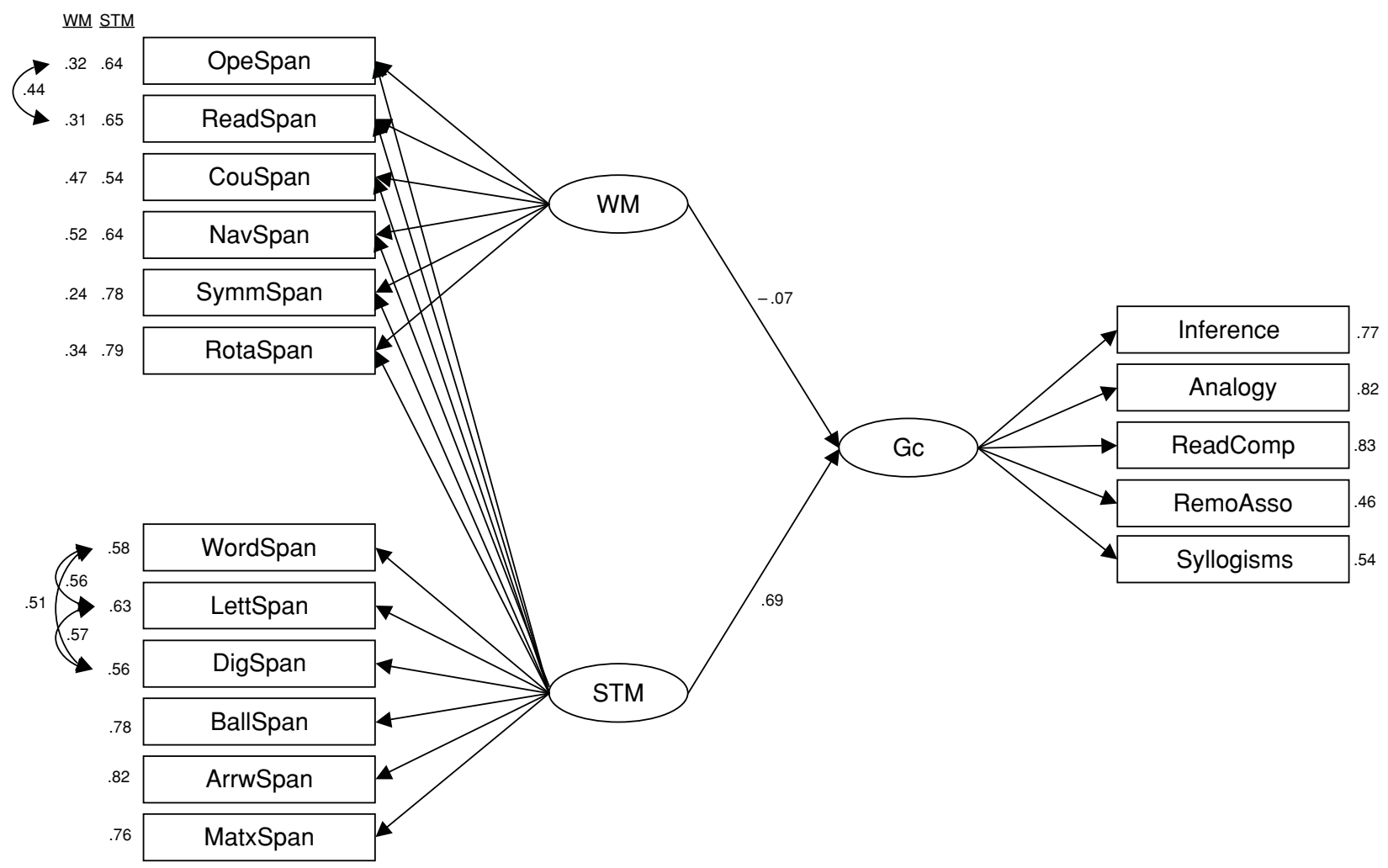

Figure 8. Structural equation modeling for crystallized intelligence (Gc) from Kane et al.'s (2004) data set, with structural coefficients. For meanings of the abbreviations, see the note to Table 6.

sidered responsible for their association with cognitive abilities.

The SEM analyses reported in the present article are consistent with this perspective. Eight of the 10 tested SEMs showed interpretable solutions. Six of them demonstrated that STM was a better predictor than WM, whereas the remaining two showed that STM and WM predicted Gf with the same power. Thus, we failed to find a data set supporting the better predictive power of WM over that of STM.

Is there a "real" sharp discrepancy between the main results derived from the original studies and those that can be extracted from the present reanalyses? We think that the latter results help to find a common ground for those studies. Engle, Tuholski, et al. (1999), Conway et al. (2002), and Kane et al. (2004) found that STM did not predict cognitive abilities, whereas Miyake et al. (2001) found that spatial STM and spatial WM are not clearly distinguishable constructs. Importantly, these four studies employed different operational approaches to answer exactly the same theoretical question. In contrast, we have subjected those different data sets to the same general model, obtaining highly consistent results across studies.

We have computed the isolated structural coefficient linking STM to the considered cognitive abilities across studies. Thus, only the latent construct representing STM was related to the latent constructs representing the con- sidered abilities. The obtained structural coefficients between STM and the various measures of cognitive ability are presented in Table 7.

It is very important to notice that the values of the structural coefficients between STM (defined by "simple" span tasks only) and the measures of the considered abilities are almost the same as the values of the coefficients reported after the SEM reanalyses in which the STM latent factor was defined by all the memory span measures. This is further evidence supporting the appropriateness of the general basic model we have endorsed in the present article (see Figure 1). Moreover, that result is inconsistent with the strong assumption held by Kane et al. (2004) - namely, that the common variance among memory span tasks reflects executive rather than storage processes. The results are consistent with the assumption held by Engle, Tuholski, et al. (1999) and by Conway et al. (2002) - namely, that the common variance among memory span tasks reflects storage rather than executive processes. However, the observed results are not consistent with Engle, Kane, and Tuholski's (1999) model (WM = STM + controlled attention). This model requires WM (with its storage component partialed out) to predict individual differences in cognitive abilities. But the results of the present reanalyses show that STM behaves as a better predictor of individual differences in cognitive abilities across data sets. 


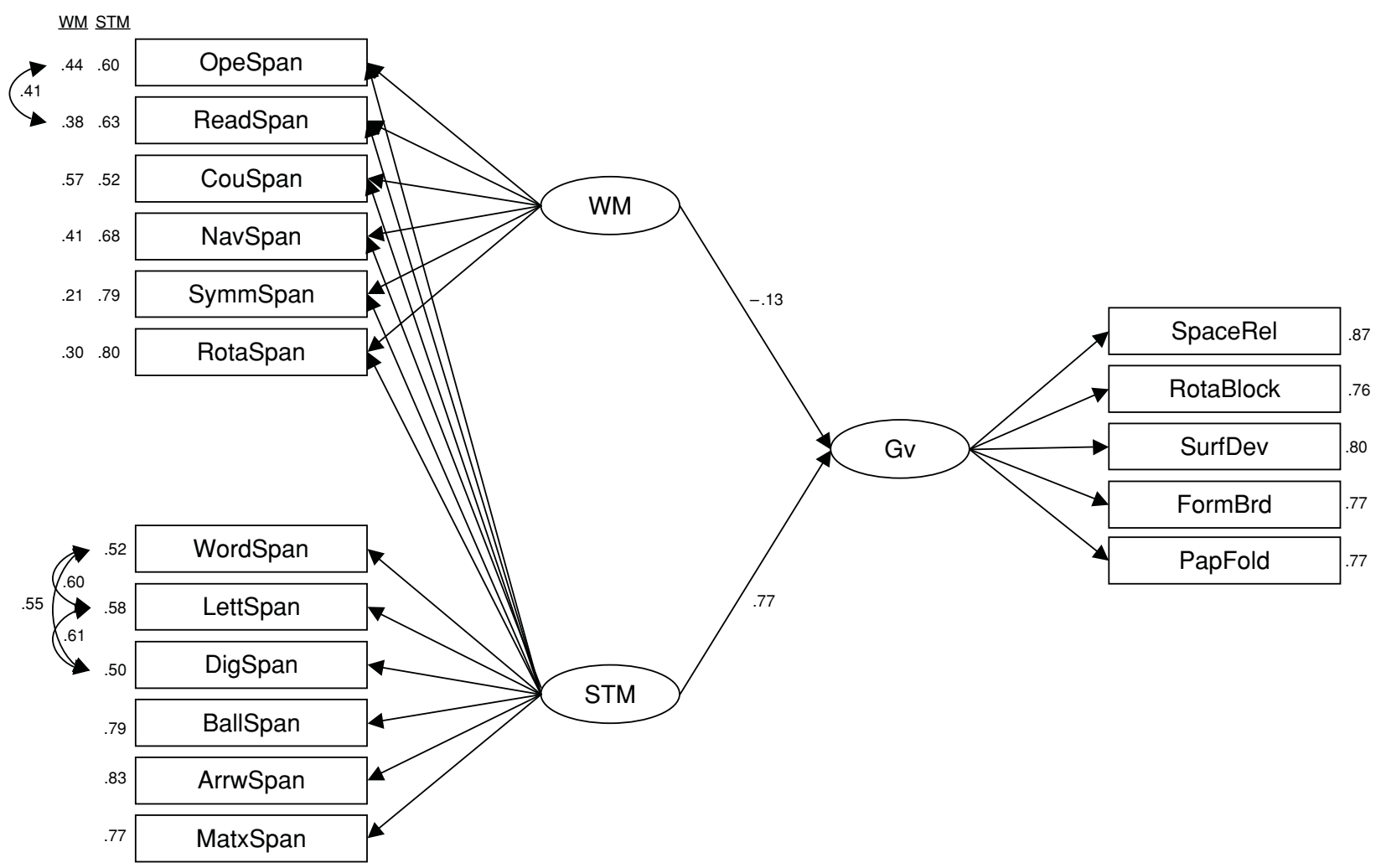

Figure 9. Structural equation modeling for spatial ability (Gv) from Kane et al.'s (2004) data set, with structural coefficients. For meanings of the abbreviations, see the note to Table 6.

Actually, STM and WM may not reflect clearly distinguishable cognitive limitations. Memory span tasks probably rely on a unitary cognitive system comprising a strong component used to temporarily preserve a reliable mental representation of information about any task. Individual differences appear to come primarily (although not exclusively) from the overall capacity and efficiency of this unitary system (Colom et al., 2004; Colom \& Shih, 2004).

The sharp difference between the tentative interpretation we endorse and the controlled attention model is the

Table 7

Obtained Structural Coefficients Between STM and Cognitive Abilities

\begin{tabular}{lcc}
\hline \multicolumn{1}{c}{ Data Set } & Between STM and: & $\begin{array}{c}\text { Obtained Structural } \\
\text { Coefficient }\end{array}$ \\
\hline Engle et al. (1999) & Gf & .26 \\
Miyake et al. (2001) & Gc & .64 \\
Conway et al. (2002) & SR & .59 \\
Kane et al. (2004) & GS & .68 \\
& Gf & .19 \\
& Gc & .82 \\
& Gv & .67 \\
\hline
\end{tabular}

Note-Gf, fluid intelligence; Gc, crystallized intelligence; SR, spatial relations; PS, perceptual speed; Gv, spatial ability. difference between the number of elements that can be temporarily retained in a reliable state on one hand, and the presumed control processes dedicated to keeping a representation active in the face of a different concurrent requirement on the other. The first interpretation relies on the concept of overall capacity rather than on that of a problem of interference between two concurrent activities.

STM measures do not need dedicated components to select any particular relevant information, mainly because all the information is relevant. Thus, for instance, single letters are presented at the rate of $1 / \mathrm{sec}$ and the participant is allowed unlimited time to type, in direct order, the letters presented. Little or no switch of attention from the representation of the letters is required. The task simply requires the temporary maintenance of the letters.

However, WM measures could require control processes because they impose dual task demands. The participant could be required to perform a verification task and a recall task. Thus, for instance, $6 \mathrm{sec}$ are allowed for verifying the accuracy of several math equations, and the participant is instructed to remember the displayed solutions irrespective of their accuracy. After the final equation of a given trial, the participant must remember each of the presented solutions in the correct serial order. These WM measures require that the target items (i.e., the displayed solutions) be maintained while concurrent cogni- 
tive processing (i.e., the verification of the math equations) is performed.

It is hardly questionable that the WM system is devoted to keeping memory representations active, accessible, and reliable through its available capacity. Cowan (2004) stated that the main difference between STM and WM measures could be the benefit of rehearsal in STM measures. In WM measures, the concurrent processing component precludes rehearsal. Therefore, there appears to be a general component for maintaining any information in an active and reliable state. Kane et al. (2004) suggested that this component must be identified with one presumed controlled attention ability, although we suggest that the evidence is more consistent with the view that this component has little to do with this attention ability.

We find it hard to believe that the temporary maintenance of reliable memory representation is not heavily influenced by the total efficiency of the WM system. Participant A and Participant B can pay attention to a given math equation while retaining the displayed solution, but why does Participant A manage to process that information whereas Participant B fails to do so? We suggest that the difference lies in the fact that Participant A enjoys more capacity than Participant B. The additional processing requirements lower the reliability of the temporary retention of information. Those requirements take a given amount of capacity away from the temporary maintenance of the relevant information. The main source of individual differences comes from a general capacity rather than from a presumed attentional ability.

In a seminal study, Carroll (1991) reanalyzed 38 cognitive ability tests and cognitive tasks considered by Kranzler and Jensen (1991). Carroll (1991) wrote, "Kranzler and Jensen did not take advantage of the opportunity their data offered to make a complete factorial analysis of all variables, as seemed to me more desirable" (p. 428). He found that cognitive tasks tend to fall together with scores on the cognitive ability battery. He thought that the identified higher order factor "can be interpreted as one [factor] measuring efficiency in complex information processing" (p. 435, emphasis added). The parsimonious interpretation we endorse suggests that there are cognitive systems more prone to coping successfully with several different challenges. There are great individual differences in the ability to efficiently process any given information.

Interestingly, Oberauer, Lange, and Engle (2004) have recently written,

We might regard simple span tasks as the more complex measures, in that they reflect more of a mixture of different sources of variance. Whereas the starting point of our work was the equation "complex span = simple span + controlled attention" (Engle[, Tuholski,] et al., 1999), it might be more fruitful to turn things around: Simple span = complex span + specialized mechanisms or strategies. (p. 94)

Their results did not support theories identifying WM with the ability to resist interference or the ability to coordinate two concurrent tasks. Furthermore, their results suggest that the difference between complex and simple span tasks cannot be interpreted as measuring the added contribution of a general executive device. The unique predictive power of complex span tasks cannot be attributed to general executive attention:

Several promising current ideas about the nature of socalled complex span tasks might have to be rethought. ... [O]ur data should at least motivate proponents of the interference account of WM (including ourselves) and proponents of the central executive account to specify more precisely under which conditions the amount of dual task interference should reflect WM (or the capacity of the central executive). (Oberauer et al., 2004, p. 93)

In summary, the findings reported here are consistent with the view that there is one general capacity explaining a great amount of the observed individual differences in both memory span tasks and cognitive ability tasks. Tasks requiring more capacity are generally those identified as WM or complex span tasks. This has led several researchers to propose that something that is not measured by socalled simple span (or STM) tasks must be responsible for the observed correlation between complex span (or WM) tasks and cognitive abilities. However, the present findings call for a reexamination of that perspective. Concurrent processing requirements leave less capacity for temporary storage of information. This diminishes the reliability of the stored information, which in turn is responsible for the behavioral effects observed in memory span tasks.

\section{REFERENCES}

Ackerman, P. L., Beier, M. E., \& Boyle, M. O. (2002). Individual differences in working memory within a nomological network of cognitive and perceptual speed abilities. Journal of Experimental Psychology: General, 131, 567-589.

Ackerman, P. L., Beier, M. E., \& Boyle, M. O. (2005). Working memory and intelligence: The same or different constructs? Psychological Bulletin, 131, 30-60.

ARBUCKLE, J. L. (2003). AMOS 5 [Computer software]. Chicago: SmallWaters.

Bayliss, D. M., Jarrold, C., GunN, D. M., \& Baddeley, A. D. (2003). The complexities of complex span: Explaining individual differences in working memory in children and adults. Journal of Experimental Psychology: General, 132, 71-92.

BYRNe, B. M. (1998). Structural equation modeling with LISREL, PRELIS, and SIMPLIS: Basic concepts, applications, and programming. Mahwah, NJ: Erlbaum.

CARroll, J. B. (1991). No demonstration that $g$ is not unitary, but there's more to the story: Comment on Kranzler and Jensen. Intelligence, 15, 423-436.

CARroll, J. B. (1993). Human cognitive abilities: A survey of factoranalytic studies. Cambridge: Cambridge University Press.

Colom, R., Abad, F. J., Rebollo, I., \& Shih, P. C. (2005). Memory span and general intelligence: A latent-variable approach. Intelligence, 33, 623-642.

Colom, R., Flores-Mendoza, C., \& Rebollo, I. (2003). Working memory and intelligence. Personality \& Individual Differences, 34, 33-39.

Colom, R., Rebollo, I., Palacios, A., Juan-Espinosa, M., \& KylLONEN, P. (2004). Working memory is (almost) perfectly predicted by g. Intelligence, $\mathbf{3 2}, 277-296$.

CoLOM, R., \& SHIH, P. C. (2004). Is working memory fractionated onto different components of intelligence? A reply to Mackintosh and Bennett (2003). Intelligence, 32, 431-444. 
Conway, A. R. A., Cowan, N., Bunting, M. F., Therriault, D. J., \& MinkofF, S. R. B. (2002). A latent variable analysis of working memory capacity, short-term memory capacity, processing speed, and general fluid intelligence. Intelligence, 30, 163-184.

CowAN, N. (2004). Working memory capacity limits in a theoretical context. In C. Izawa \& N. Ohta (Eds.), Human learning and memory: Advances in theory and application (pp. 155-175). Mahwah, NJ: Erlbaum.

DetTerman, D. K. (1989). The future of intelligence research. Intelligence, 13, 199-203.

EMBRETSON, S. (1995). The role of working memory capacity and general control processes in intelligence. Intelligence, 20, 169-189.

ENGLE, R. W., \& KANE, M. J. (2004). Executive attention, working memory capacity, and a two-factor theory of cognitive control. In B. H. Ross (Ed.), The psychology of learning and motivation: Advances in research and theory (Vol. 44, pp. 145-199). New York: Elsevier.

Engle, R. W., Kane, M. J., \& Tuholski, S. W. (1999). Individual differences in working memory capacity and what they tell us about controlled attention, general fluid intelligence, and functions of the prefrontal cortex. In A. Miyake \& P. Shah (Eds.), Models of working memory (pp. 102-134). New York: Cambridge University Press.

Engle, R. W., Tuholski, S. W., Laughlin, J. E., \& Conway, A. R. A. (1999). Working memory, short-term memory, and general fluid intelligence: A latent-variable approach. Journal of Experimental Psychology: General, 128, 309-331.

Friedman, N. P., \& MiYaKe, A. (2000). Differential roles for visuospatial and verbal working memory in situation model construction Journal of Experimental Psychology: General, 129, 61-83.

Jensen, A. (1998). The g factor. Westport: Praeger.

Jensen, A., \& Weng, L. (1994). What is a good g? Intelligence, 18, 231-258.

JöRESKOG, K. G. (1993). Testing structural equation models. In K. A. Bollen \& J. S. Long (Eds.), Testing structural equation models (pp. 294-316). Newbury Park, CA: Sage.

Kane, M. J., Hambrick, D. Z., Tuholski, S. W., Wilhelm, O., Payne, T. W., \& ENGLE, R. W. (2004). The generality of working memory capacity: A latent-variable approach to verbal and visuospatial memory span and reasoning. Journal of Experimental Psychology: General, 133, 189-217.

KuINE, P. (1994). An easy guide to factor analysis. London: Routledge.

Kranzler, J. H., \& Jensen, A. R. (1991). The nature of psychometric $g$ : Unitary process or a number of independent processes? Intelligence, 15, 397-422.
Kyllonen, P., \& Christal, R. (1990). Reasoning ability is (little more than) working memory capacity?! Intelligence, 14, 389-433.

LoEHLIN, J. C. (2004). Latent variable models: An introduction to factor, path, and structural equation analysis (4th ed.). Mahwah, NJ: Erlbaum.

Lohman, D. F. (2000). Complex information processing and intelligence. In R. J. Sternberg (Ed.), Handbook of intelligence (2nd ed., pp. 285-340). Cambridge: Cambridge University Press.

LUBINSKI, D. (2004). Introduction to the special section on cognitive abilities: 100 years after Spearman's (1904) " 'General intelligence,' objectively determined and measured." Journal of Personality \& Social Psychology, 86, 96-111.

Mackintosh, N. J., \& Bennett, E. S. (2003). The fractionation of working memory maps onto different components of intelligence. Intelligence, 31, 519-531

Marsh, H. W., Balla, J. R., \& McDonald, R. P. (1988). Goodness-offit indexes in confirmatory factor analysis: The effect of sample size. Psychological Bulletin, 103, 391-410.

MiYAKE, A. (2001). Individual differences in working memory: Introduction to the special section. Journal of Experimental Psychology: General, 130, 163-168.

Miyake, A., Friedman, N. P., Rettinger, D. A., Shah, P., \& HegARTY, M. (2001). How are visuospatial working memory, executive functioning, and spatial abilities related? A latent-variable analysis. Journal of Experimental Psychology: General, 130, 621-640.

Oberauer, K., Lange, E., \& Engle, R. W. (2004). Working memory capacity and resistance to interference. Journal of Memory \& Language, 51, 80-96.

Salthouse, T. A., Atkinson, T. M., \& Berish, D. E. (2003). Executive functioning as a potential mediator of age-related cognitive decline in normal adults. Journal of Experimental Psychology: General, 132, 566-594.

Schmid, J., \& Leiman, J. M. (1957). The development of hierarchical factor solutions. Psychometrika, 22, 53-61.

Süß, H.-M., Oberauer, K., Wittmann, W. W., Wilhelm, O., \& Schulze, R. (2002). Working memory capacity explains reasoning ability — and a little bit more. Intelligence, 30, 261-288.

TURNER, M. L., \& ENGLE, R. W. (1989). Is working memory capacity task dependent? Journal of Memory \& Language, 28, 127-154.

(Manuscript received March 22, 2004; revision accepted for publication January 7, 2005.) 